\title{
CORRECTIONS
}

\section{Author Correction: The role of sex in the genomics of human complex traits}

\author{
Ekaterina A. Khramtsova, Lea K. Davis and Barbara E. Stranger
}

Nature Reviews Genetics (2019) https://doi.org/10.1038/s41576-018-0083-1

Published online 23 December 2018

In Box 4 of the originally published article, the text describing the Miami plot in part c of the figure contained some minor errors. Specifically, during pre-publication revision of the article, the authors updated the illustrative Miami plot (shown in figure part c) from that of reference 80 (Randall et al. PLoS Genet. (2013)) to a more recent study in reference 82 (Winkler et al. PLoS Genet. (2015)). The box text has now been updated to reflect that change. In paragraph 2, the trait has been updated from "hip circumference adjusted for body mass index" to "waist-to-hip ratio adjusted for body mass index (under 50 years old)" and it has been clarified that female GWAS data are shown on the top half of the plot with male data at the bottom. The original two citations of reference 80 in the Box 4 text have been updated to reference 82 , and the annotation of reference 82 in the article reference list has been updated to state that it is a follow-up study to reference 80 . Finally, a typographical artefact was corrected on the $Y$ axis of the Miami plot, whereby the labels ' 14 ' and ' 16 ' in the top half of the plot we originally both shown as ' 12 '. None of these corrections alter the overall illustrative point that genetic architectures for traits can differ between males and females, which was a conclusion of both reference 80 and reference 82 .

https://doi.org/10.1038/s41576-019-0148-9 I Published online 28 June 2019 